2012

\title{
On estimating the precision of stable isotope ratios in processed tree-rings
}

Post-print/Accepted manuscript

Trevor J. Porter

Paul Middlestead

Porter, T.J., Middlestead, P., 2012. On estimating the precision of stable isotope ratios in processed tree-rings. Dendrochronologia 30, 239-242. doi:10.1016/i.dendro.2012.02.001

\section{HOW TO CITE TSPACE ITEMS}

Always cite the published version, so the author(s) will receive recognition through services that track citation counts, e.g. Scopus. If you need to cite the page number of the TSpace version (original manuscript or accepted manuscript) because you cannot access the published version, then cite the TSpace version in addition to the published version using the permanent URI (handle) found on the record page. 
1 On estimating the precision of stable isotope ratios in processed tree-rings

3 Trevor J. Porter ${ }^{1, *}$ and Paul Middlestead ${ }^{2}$

$4 \quad{ }^{1}$ Department of Geography and Environmental Studies, Carleton University, 1125 Colonel By

5 Drive, Ottawa, Canada, K1S5B6

$6{ }^{2}$ G.G. Hatch Stable Isotope Laboratory, University of Ottawa, 130 Louis-Pasteur Private,

7 Ottawa, Canada, K1N6N5

8

9 *Corresponding author: +1-613-520-2600 ext.1836 (office); +1-613-520-4301 (fax);

10 tjporter@connect.carleton.ca

11

12 Keywords: tree-rings; stable isotope ratios; cellulose; precision; quality assurance standard;

13 identical treatment

14

15

16

17

18 


\section{Abstract}

Stable isotope dendrochronology is a well-developed field of research, but improvements to methodologies are on-going. We propose an improved method for estimating the precision of

22 stable isotope ratios $(\delta)$ of tree-ring samples that are processed from whole wood to various end

23 products such as cellulose-nitrate, $\alpha$-cellulose, or cellulose intermediates. The status quo method

24 for estimating the $\delta$ precision of organic solids is to characterize the long-term 2-sigma range of

$25 \delta$ values for a ready-made Quality Assurance (QA) standard that is included in each analysis run

26 of samples. While the status quo method is appropriate for characterizing analytical uncertainties

27 associated with the mass spectrometer, combustion or pyrolysis system, and analyte specifics, it

28 does not reflect uncertainties associated with sample processing from inadvertent and unrealised

29 operator error (e.g., contamination by airborne particles, incomplete chemical processing, sample

30 storage issues, and other unforeseen errors), although such errors would probably be rare with an

31 experienced operator. The proposed method improves upon the status quo method as it respects

32 the Identical Treatment principle by subjecting QA standards to the same processing steps that

33 samples undergo. As such, analytical uncertainties associated with sample processing would be

34 integrated into the QA standard's $\delta$ value and precision estimate. In effect, the proposed method

35 is a system to monitor inter-batch reproducibility and, by the same token, can be used to identify

36 batches that were potentially compromised during processing. A pilot study example is used to

37 demonstrate the proposed method for $\delta^{18} \mathrm{O}$ analysis of $\alpha$-cellulose samples. 


\section{Introduction}

42

43

44

45

46

47

48

49

50

51

52

53

54

60

61

62

Stable isotope ratios $(\delta)$ in tree-rings are a valuable resource for dendrochronologists addressing both climatic (e.g., Treydte et al., 2006; Porter et al., 2009) and ecophysiological (e.g., Feng, 1999; Waterhouse et al., 2004) questions. Since stable isotope dendrochronology was first demonstrated (Craig, 1954), standard methods have been adopted and improved over the years (McCarroll and Loader, 2004), and continue to be refined even today (e.g., Laumer et al., 2009; Gagen et al., 2012). Here, we focus on the reproducibility or precision of tree-ring $\delta$ values and, specifically, how it is estimated for samples processed from whole wood to various end products such as cellulose-nitrate, $\alpha$-cellulose, or cellulose intermediates; we use the term 'processed tree-rings' to refer to such samples. This discussion applies mainly to stable isotopes that vary between whole wood and processed tree-rings due to isotopic biases of various wood compounds (i.e., $\delta^{13} \mathrm{C}$ ), or exchange of loosely-bound $\mathrm{H} / \mathrm{O}$ atoms with ambient $\mathrm{H}_{2} \mathrm{O}$ or adhesion of ambient $\mathrm{H}_{2} \mathrm{O}$ (i.e., $\delta^{2} \mathrm{H} / \delta^{18} \mathrm{O}$ ). We propose a standard method for estimating the $\delta$ precision of processed tree-rings that accounts for analytical uncertainties associated with sample processing. To our knowledge, many tree-ring isotope studies do not control for such uncertainties, or report that they do.

\section{Background}

Stable isotope ratio measurements are subject to analytical uncertainties associated with the mass spectrometer, combustion or pyrolysis system, and sample amount and purity (Werner and Brand, 2001). These uncertainties lead to variability in measured $\delta$ values of samples which can be significant compared to variability between tree-rings and, thus, it is standard practice to 
report the $2 \sigma$ (2 standard error) precision. Ceteris paribus, $\delta$ precisions of ca. $\pm 2.5 \%$, $\pm 0.1 \%$, and $\pm 0.3 \%$ are typical for continuous-flow analysis of wood $\delta^{2} \mathrm{H}, \delta^{13} \mathrm{C}$, and $\delta^{18} \mathrm{O}$, respectively (McCarroll and Loader, 2004). Typically, $\delta$ precision is estimated from a long-term, running standard deviation of $\delta$ values measured for a Quality Assurance (QA) standard analysed along with each batch of samples introduced to the mass spectrometer; $\delta$ values for samples and the QA standard are calibrated against 2 or more internal reference standards that are also analysed along with each batch of samples, and whose $\delta$ values with respect to an internationally-accepted reference scale (e.g., VSMOW or VPDB) are well known. Ideally, the samples, QA standard, and reference standards are all of the same matrix (e.g., $\alpha$-cellulose; Boettger et al., 2007; Brand et al., 2009).

For routine $\delta$ analysis of processed tree-rings, bulk quantities of the reference standards and QA standard are typically purchased commercially or developed in-house from whole wood using the same processing method applied to samples, consistent with the Identical Treatment or 'IT' principle (Werner and Brand, 2001). However, in the case of the QA standard, we caution against the use of such 'ready-made' QA standards as they do not reflect uncertainties associated with sample processing that can impact upon the purity and $\delta$ of samples, mainly operator-related errors. Operator errors are inadvertent and unrealised, for if they were realised the compromised samples should not be analysed. These errors may include, but are not limited to, contamination from airborne particulates, processing irregularities (e.g., reactions were not always carried out to completion due to insufficient/variable solution strengths, heating, and reaction times; inadequate rinsing of chemical residues; etc.), and insufficient desiccation prior to $\delta^{2} \mathrm{H} / \delta^{18} \mathrm{O}$ analysis. Such errors are likely rare for an experienced operator. However, many tree-ring $\delta$ projects are carried out by senior undergraduate and graduate students, some of whom are learning these methods for 
the first time and may have limited laboratory experience. Operator error may occur more often

87 in such cases, and the proposed method provides a system to monitor inter-batch reproducibility and identify potentially compromised results.

\section{Proposed method}

To estimate the $\delta$ precision of processed tree-rings, the IT principle must be respected by

92 exposing the QA standard to the same chemical processing steps that sample tree-rings undergo. Ideally, a bulk reserve (e.g., enough for several years of analysis) of homogeneous whole wood

94 particles, of roughly the same consistency as a sample, should be prepared by milling a piece of wood into smaller particles and used as the QA standard. Alternatively, bulk quantities of wood flour or shavings can be purchased commercially. Aliquots of the QA standard, consistent in mass to a typical sample, should be processed along with each batch of samples so that the QA standard is exposed to the same potential errors a sample is exposed to, which would be reflected 99 in its $\delta$ value and the overall precision estimate.

To ensure that $\delta$ variability in the processed QA standard is not influenced by sampling error, it is critical that all aliquots are isotopically representative of the bulk reserve from which they were sampled. For large or small aliquots, a representative aliquot can be assured if the QA standard is a homogeneous mixture of fine-grained particles, e.g., approaching the consistency of

104 fine sand. However, fine-grained particles are only appropriate for cellulose extraction methods 105 that process samples individually in test tubes (e.g., Brendel et al., 2000). For 'batch-processing' methods that require samples to be cut into coarse-granules or slivers (e.g., Leavitt and Danzer,

107 1993; Loader et al., 1997), a coarse-grained QA standard should be used. Granular particles are 
ideal to facilitate a well-mixed bulk reserve. To avoid homogeneity issues with a coarse-grained QA standard, larger aliquots should be taken since individual granules may have a wide range of $\delta$ values; experimentation may be needed to determine the appropriate aliquot size.

During processing, all reasonable efforts should be exercised to ensure that samples and QA standards are treated equally (i.e., all reactions carried out to completion; samples and QA standards are rotated in the heating block or bath; etc.) to avoid potential systematic differences in the purity and $\delta$ of samples and QA standards. Once processing is complete, samples and QA standards should be sub-sampled for $\delta$ analysis. Sub-sampling is required to ensure the amount of sample, or QA standard, introduced to the mass spectrometer system is constant as variability in sample amount can contribute variability to measured $\delta$ values. Fine-grained samples and QA standards can be readily sub-sampled as they are homogeneous, while coarse-grained materials must first be homogenised by mechanical milling or ultrasonic breaking (Laumer et al., 2009).

Precision can be estimated by characterising the $2 \sigma$ range of a given series of $\delta$ values for the QA standard, although the number of QA data points included in the estimate will vary from one laboratory to the next based on internal protocols and, of course, the purpose of the estimate. In any case, there is no universal number of QA data points that must be used, as $\delta$ precision is a statistical estimate that accounts for sample size, but we recommend Bessel's correction (degrees of freedom $=\mathrm{n}-1$ ) is used to err on the side of caution, especially with small sample sizes. If the purpose of the estimate is to quantify long-term $\delta$ reproducibility for a given laboratory, then the estimate would likely contain hundreds to thousands of QA data points that span several years of routine analysis. However, if the purpose is to quantify $\delta$ reproducibility associated with a given project (e.g., tree-ring series), then it is more appropriate to use only QA data points from that project. Regardless, $\delta$ precision reporting should be unambiguous by stating whether or not the 
131 QA standard was processed along with samples, if the estimate is project-specific or a long-term 132 average for the laboratory that conducted the analysis, and how many QA data points were used.

Lastly, it should be noted that the proposed method provides an opportunity to monitor

134 inter-batch $\delta$ reproducibility and identify batches that have potentially been compromised during 135 sample processing, which may be of considerable value in cases where inexperienced operators

136 are involved. For example, if the $\delta$ value for a QA standard falls outside the acceptable range of $137 \delta$ values (as per internal laboratory protocols; e.g., 99\% confidence interval), then the batch that 138 the QA standard belonged to should be scrutinised. If upon a second analysis the QA standard $\delta$ 139 value is still found to be an outlier, the entire batch should be reprocessed or excluded. The final 140 course of action, however, should be dictated by internal laboratory protocols.

\section{Pilot study}

The proposed method was used in a recent tree-ring $\delta^{18} \mathrm{O}$ project carried out at the G.G.

144 Hatch Stable Isotope Laboratory. The objective was to measure the $\delta^{18} \mathrm{O}$ of 210 tree-rings from 145 white spruce (Picea glauca [Moench] Voss) trees sampled at 'Timber' site (Porter et al., 2009) 146 near boreal treeline in the Mackenzie Delta, northern Canada. For our QA standard, we 147 developed a homogeneous mixture of fine-grained wood particles from a large diameter core 148 collected from the study site. The samples and the QA standard were homogenised using a 149 Retsch-Brinkmann MM200 Mixer Mill. The 210 samples plus 14 QA standard aliquots (ca. 70 $150 \mathrm{mg}$ ) were processed in batches of 16 over 14 days. Each batch was processed from whole wood 151 to $\alpha$-cellulose in a single day using the rapid extraction method outlined by Brendel et al. (2000), 152 but modified to include an alkaline hydrolysis step as suggested by Gaudinski et al. (2005). 
After processing, the samples and QA standards were placed in a drying oven at $50^{\circ} \mathrm{C}$ overnight, and then stored in a vacuum-sealed desiccator with Drierite, a gypsum-based desiccant.

Samples and QA standards were weighed (ca. 150-250 $\mu \mathrm{g}$ material) into silver capsules, returned to the desiccator for a minimum of 24 hours, and then loaded into a Costech zero-blank auto-sampler carousel. The auto-sampler was put under vacuum and then purged with dry helium for 5 minutes before the $\delta^{18} \mathrm{O}$ analysis began. A Thermo-Finnigan TC-EA and DeltaXP IRMS were used for the analysis (system parameters: pyrolysis tube at $1400^{\circ} \mathrm{C} ; 5 \AA$ GC column at $90^{\circ} \mathrm{C} ; 100 \mathrm{~mL} / \mathrm{min}$ flow rate). Samples and QA standards were analysed in the order they were processed. Each QA $\delta^{18} \mathrm{O}$ value reported here (Table 1) represents a single pyrolysis. Sample and QA $\delta^{18} \mathrm{O}$ values were calibrated using $3 \alpha$-cellulose reference standards with $\delta^{18}$ Ovsmow values of $20.3,27.5$, and $32.8 \%$. Ideally, the $\delta^{18} \mathrm{O}$ range of the reference standards should bracket the $\delta^{18} \mathrm{O}$ values of the unknown samples; however, our QA standard (whose $\delta^{18} \mathrm{O}$ was unknown before the analysis) was found to be depleted by ca. 3\%o compared to our most negative reference standard (i.e., 20.3\%o). However, this is understandable since tree-rings in the Mackenzie Delta are labelled by some of the most isotopically-depleted precipitation in the Northern Hemisphere (Bowen and Revenaugh, 2003), and there are few sources of natural wood cellulose as depleted, high-Arctic shrubs being an exception (Welker et al., 2005). Nevertheless, the 3-point calibration curve we used was quite linear $\left(\mathrm{r}^{2} \geq 0.99\right)$ over the range $20.3-32.8 \%$, and extrapolation by ca. $3 \%$ is reasonable.

The 14 QA standards had a $\delta^{18} \mathrm{O}_{\text {VsMow }}$ range of $17.1-17.5 \%$, a mean of $17.3 \%$, and a $2 \sigma$ precision of $0.3 \%$ (Table 1 ). As discussed above, a precision of $0.3 \%$ is typical for continuousflow analysis of tree-ring $\delta^{18} \mathrm{O}$, suggesting that the samples and QA standards were consistently 
175 processed to the same end product and probably did not accumulate much error in excess of what

176

177 can be explained by uncertainties associated with the mass spectrometer, pyrolysis environment, and specifics of $\alpha$-cellulose.

However, had we not been so careful in treating all samples and QA standards the same, the $\delta$ precision estimate could have been very different. To demonstrate this point, 2 additional QA aliquots were prepared to $\alpha$-cellulose and purposely not desiccated prior to analysis. As $\alpha$ cellulose is a hygroscopic compound, it was expected that exposure to ambient humidity would contribute error to the $\delta^{18} \mathrm{O}$ value. The 2 purposely-compromised QA standards were analysed on 2 separate days, both having a $\delta^{18} \mathrm{O}$ value of $18.2 \%$. This compromised $\delta^{18} \mathrm{O}$ value is greater than $+7 \sigma$ from the mean of the desiccated QA standards, thereby demonstrating how improper storage of samples, or even a leak in the desiccator, could lead to error in the $\delta^{18} \mathrm{O}$ of samples. If such an error did occur during routine analysis, and the QA standard was not processed using the proposed method, it might not be possible to identify the error post facto.

\section{Conclusions}

There are many sources of uncertainty that could influence the $\delta$ of processed tree-rings before samples are introduced to the mass spectrometer. To better characterize the $\delta$ precision of processed samples, QA standards must be treated as if they are samples. In isotope laboratories that specialize in tree-ring analysis and offer the service of preparing tree-ring cellulose, their inhouse protocols could be revised fairly easily. However, in cases where the tree-ring researcher is responsible for preparing their own samples before sending them to an isotope laboratory, the onus is on the researcher to provide the QA standards. In such cases, the isotope laboratory may 
recognize the merit of using the proposed method and waive the costs associated with analyzing additional QA standards. Again, the onus is on the researcher to inquire about such details.

\section{Acknowledgements}

$$
\text { assistance. We thank M. Saurer and one anonymous reviewer for their constructive comments }
$$
which led to a much improved paper. This work also benefitted from an NSERC postgraduate scholarship to T. Porter.

\section{References}

Boettger, T., Haupt, M., Knöller, K., Weise, S.M., Waterhouse, J.S., Rinne, K.T., Loader, N.J., Sonninen, E., Jungner, H., Masson-Delmotte, V., Stievenard, M., Guillemin, M.-T., Pierre, M., Pazdur, A., Leuenberger, M., Filot, M., Saurer, M., Reynolds, C.E., Helle, G., Schleser, G.H., 2007. Wood cellulose preparation methods and mass spectrometric analyses of $\delta^{13} \mathrm{C}, \delta^{18} \mathrm{O}$, and nonexchangeable $\delta^{2} \mathrm{H}$ values in cellulose, sugar, and starch: an interlaboratory comparison. Analytical Chemistry 79, 4603-12.

Bowen, G.J., Revenaugh, J., 2003. Interpolating the isotopic composition of modern meteoric precipitation. Water Resources Research 39, doi:10.1029/2003WR002086.

Brand, W.A., Coplen, T.B., Aerts-Bijma, A.T., Böhlke, J.K., Gehre, M., Geilmann, H., Gröning, M., Jansen, H.G., Meijer, H.A.J., Mroczkowski, S.J., Qi, H., Soergel, K., Stuart-Williams, H., Weise, S.M., Werner, R.A., 2009. Comprehensive inter-laboratory calibration of reference materials for $\delta^{18} \mathrm{O}$ versus VSMOW using various on-line high-temperature conversion techniques. Rapid Communications in Mass Spectrometry 23, 999-1019.

Brendel, O., Iannetta, P.P.M., Stewart, D., 2000. A rapid and simple method to isolate pure alpha-cellulose. Phytochemical Analysis 11, 7-10.

Craig, H., 1954. Carbon-13 variations in Sequoia rings and the atmosphere. Science 119, 14143. 
Feng, X., 1999. Trends in intrinsic water-use efficiency of natural trees for the past 100-200 years: A response to atmospheric $\mathrm{CO}_{2}$ concentration. Geochimica et Cosmochimica Acta 63, 1891-1903.

Gagen, M., McCarroll, D., Jalkanen, R., Loader, N.J., Robertson, I., Young, G.H.F., 2012. A rapid method for the production of robust millennial length stable isotope tree ring series for climate reconstruction. Global and Planetary Change 82-83, 96-103.

Gaudinski, J.B., Dawson, T.E., Quideau, S., Schuur, E.A.G., Roden, J.S., Trumbore, S.E., Sandquist, D.R., Oh, S.-W., Wasylishen, R.E., 2005. Comparative analysis of cellulose preparation techniques for use with ${ }^{13} \mathrm{C},{ }^{14} \mathrm{C}$, and ${ }^{18} \mathrm{O}$ isotopic measurements. Analytical Chemistry 77, 7212-24.

Laumer, W., Andreu, L., Helle, G., Schleser, G.H., Wieloch, T., Wissel, H., 2009. A novel approach for the homogenization of cellulose to use micro-amounts for stable isotope analyses. Rapid Communications in Mass Spectrometry 23, 1934-40.

Leavitt, S.W., Danzer, S.R., 1993. Method for batch processing small wood samples to holocellulose for stable-carbon isotope analysis. Analytical Chemistry 65, 87-89.

Loader, N.J., Robertson, I., Barker, A.C., Switsur, V.R., Waterhouse, J.S., 1997. An improved technique for the batch processing of small wholewood samples to $\alpha$-cellulose. Chemical Geology 136, 313-17.

McCarroll, D., Loader, N.J., 2004. Stable isotopes in tree rings. Quaternary Science Reviews 23, 771-801.

Porter, T.J., Pisaric, M.F.J., Kokelj, S.V., Edwards, T.W.D., 2009. Climatic signals in $\delta^{13}$ C and $\delta^{18} \mathrm{O}$ of tree-rings from white spruce in the Mackenzie Delta region, northern Canada. Arctic, Antarctic, and Alpine Research 41, 497-505.

Treydte, K.S., Schleser, G.H., Helle, G., Frank, D.C., Winiger, M., Haug, G.H., Esper, J., 2006. The twentieth century was the wettest period in northern Pakistan over the past millennium. Nature 440, 1179-82.

Waterhouse, J.S., Switsur, V.R., Barker, A.C., Carter, A.H.C., Hemming, D.L., Loader, N.J., Robertson, I., 2004. Northern European trees show a progressively diminishing response to increasing atmospheric carbon dioxide concentrations. Quaternary Science Reviews 23, 803-10.

Welker, J.M., Rayback, S., Henry, G.H.R., 2005. Arctic and North Atlantic Oscillation phase changes are recorded in the isotopes $\left(\delta^{18} \mathrm{O}\right.$ and $\left.\delta^{13} \mathrm{C}\right)$ of Cassiope tetragona plants. Global Change Biology 11, 997-1002.

Werner, R.A., Brand, W.A., 2001. Referencing strategies and techniques in stable isotope ratio analysis. Rapid Communications in Mass Spectrometry 15, 501-19. 


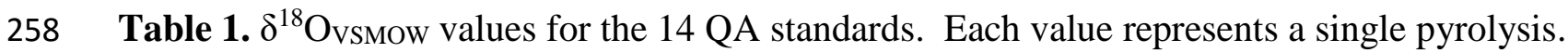

259 The analysis took place over several weeks from July 12 to August 30, 2010.

\begin{tabular}{ccc}
\hline QA std. & $\delta^{18}$ O (\%) & Date \\
\hline W01 & 17.5 & Jul-12 \\
W02 & 17.1 & Jul-12 \\
W03 & 17.4 & Jul-14 \\
W04 & 17.5 & Aug-13 \\
W05 & 17.5 & Aug-16 \\
W06 & 17.2 & Aug-17 \\
W07 & 17.4 & Aug-18 \\
W08 & 17.1 & Aug-18 \\
W09 & 17.3 & Aug-24 \\
W10 & 17.5 & Aug-25 \\
W11 & 17.4 & Aug-26 \\
W12 & 17.2 & Aug-27 \\
W13 & 17.4 & Aug-30 \\
W14 & 17.2 & Aug-30 \\
\hline Mean (W01-W14) $=17.3 \% ; 2 \sigma=0.3 \%$
\end{tabular}

